PROCEEDINGS OF THE

AMERICAN MATHEMATICAL SOCIETY

Volume 75, Number 1, June 1979

\title{
MEASURES WHOSE INTEGRAL TRANSFORMS ARE PLURIHARMONIC
}

\author{
CLINTON KOLASKI
}

AbSTRaCt. Kolaski proved an F. and M. Riesz type theorem for the unit ball in $\mathbf{C}^{N}$. This paper generalizes those results.

1.1. Introduction. Throughout this paper the complex field shall be denoted by $\mathrm{C}$, the nonnegative integers by $Z_{+}$and the unit circle by $T: \mathrm{C}^{N}, Z_{+}^{N}$ and $T^{N}$ shall denote the Cartesian product of $N$ copies of $\mathrm{C}, Z_{+}$and $T$, respectively.

For $(z, w) \in \mathbf{C}^{N} \times \mathbf{C}^{N}, \alpha \in Z_{+}^{N}$ we define $z^{\alpha}=z_{1}^{\alpha_{1}} z_{2}^{\alpha_{2}} \cdots z_{N}^{\alpha_{N}},|\alpha|=\alpha_{1}+$ $\alpha_{2}+\cdots+\alpha_{N}$ and $\alpha !=\left(\alpha_{1} !\right)\left(\alpha_{2} !\right) \cdots\left(\alpha_{N} !\right)$.

$\mathbf{C}^{N}$ shall be topologized by the inner product $\langle z, w\rangle=z \bar{w}+\cdots+z_{N} \bar{w}_{N}$; as is usual, we set $|z|=\langle z, z\rangle^{1 / 2}$.

The open unit ball in $\mathbf{C}^{N}$ is denoted by $B$; for $N=1$, its boundary is $T$, for $N \geqslant 2$, its boundary is the sphere $S=\{|z|=1\}$.

We shall let $m_{T}, m_{S}$ and $m_{B}$ denote Lebesgue measure on $T, S$ and $B$, respectively, all normalized to have unit mass.

Given a (locally compact) subset $E$ of $\mathbf{C}^{N}, M(E)$ shall denote the (Banach) space of bounded Borel measures on $E$.

A subset $E$ of $\mathbf{C}^{N}$ shall be called $T\left[T^{N}\right]$ invariant providing $\omega z \in E$ whenever $\omega \in T\left[T^{N}\right]$ and $z \in E$ (if $(\omega, z) \in T^{N} \times E$, then $\omega z=$ $\left.\left(\omega_{1} z_{1}, \ldots, \omega_{N} z_{N}\right)\right)$. A measure $\sigma \in M(E)$ is called $T\left[T^{N}\right]$ invariant providing $\sigma(\omega A)=\sigma(A)$ for all $\omega$ in $T\left[T^{N}\right]$ and all Borel subsets $A$ of $E$. Equivalently, $\int f(\omega z) d \sigma(z)=\int f(z) d \sigma(z)$ for all $\omega$ in $T\left[T^{N}\right]$ and all $f$ in $C_{0}(E)$.

A consideration of the group of holomorphic homeomorphisms of $B$, led to the discovery of the kernels $\beta$ and $\chi$ defined by

$$
\beta(z, w)=\left[(1-\langle w, w\rangle) /|1-\langle z, w\rangle|^{2}\right]^{N}
$$

and

$$
\chi(z, w)=\left[(1-\langle w, w\rangle) /|1-\langle z, w\rangle|^{2}\right]^{N+1},
$$

for all $(z, w) \in \bar{B} \times B$.

With regards to the kernel $\beta$ there is the following question. If $u \in M(S)$, if $\int_{S} \beta(z, \cdot) d u(z)$ is pluriharmonic in $B$, and if $N \geqslant 2$, then do we have $u \ll m_{s}$ ?

Received by the editors July $18,1978$.

AMS (MOS) subject classifications (1970). Primary 43A32; Secondary 31C10, 32H10, 46G99. 
In [4] - [6], Forelli proved several results which strongly suggest that the answer is yes, but the question is still open.

In [11], it was shown that an analogous theorem holds for the kernel $\chi$. This paper generalizes [11], our main result being given by Theorem 2.2.7 which states (essentially): If $\sigma$ is a class $K$ measure with $\sigma\left(\partial \Omega_{\sigma}\right)=0$, if $u \in M\left(\Omega_{\sigma}\right)$, and if $\int_{\Omega_{\sigma}} K_{\sigma}(z, \cdot) d u(z)$ is pluriharmonic in $\Omega_{\sigma}$, then $u \ll \sigma$.

It is shown that $K_{m_{B}}$ so that our Theorem 2.2.7 includes Theorem 2.3 of [11] as a special case. It is also shown that $K_{m_{s}}=\beta$, but Theorem 2.2.7 does not answer the question posed above because the condition $\sigma\left(\partial \Omega_{\sigma}\right)=0$ fails when $\sigma=m_{S}$.

\subsection{Class $K$ measures.}

Definition 2.1.1. Given $\sigma \in M\left(C^{N}\right)$ we set $F_{\sigma}=$ Support $\sigma$ and $\Omega_{\sigma}=$ Interior Holomorphic Hull $F_{\sigma}$ (i.e., $\bar{\Omega}_{\sigma}=\left\{z \in \mathbf{C}^{N}:|f(z)| \leqslant \operatorname{Sup}_{w \in F_{\sigma}}|f(w)|\right.$, for all $f$ entire $\})$. Moreover, for $(\alpha, \beta) \in Z_{+}^{N} \times Z_{+}^{N}$ we set $\hat{\sigma}(\alpha, \beta)=$ $\int z^{\alpha} \bar{z}^{\beta} d \sigma(z)$.

Definition 2.1.2. Let $\sigma \neq 0$ be a nonnegative, $T^{N}$-invariant measure with compact support in $\mathbf{C}^{N}$. If, in addition, $F_{\sigma} \not \subset \cup_{j=1}^{N}\left\{z_{j}=0\right\}$ (i.e., $F_{\sigma}$ is not contained in the union of the coordinate planes) then we shall call $\sigma$ a class $K$ measure.

The terminology is motivated by the fact that for each such measure, $\sigma$, there corresponds a kernel $K_{\sigma}$ such that $K_{\sigma} \cdot \sigma$ reproduces functions pluriharmonic on $\Omega_{\sigma}$ (see Definition 2.2.1 and Theorem 2.2.5).

To gain some insight into the generality of these measures, we give an example of a class $K$ measure $\sigma$ which is mutually singular with respect to $m_{B}$ even though its support is $\bar{B}$.

If $r_{1}, r_{2}, \ldots$ is an enumeration of the rationals in $(0,1)$, if $a_{1}, a_{2}, \ldots$ is a summable sequence of positive numbers, and if $\mu_{n}$ denotes normalized Lebesgue measure on the sphere $S_{n}=\left\{|z|=r_{n}\right\}$, then we set $\sigma=\sum_{n=1}^{\infty} a_{n} \cdot \mu_{n}$.

For the purpose of proving Lemma 2.1.4, we recall the following fact [9, Theorem 2.4.2].

THEOREM 2.1.3. The convergence domain $\Omega$ of a power series $\sum a_{\alpha} z^{\alpha}$ is the interior of the set $\left\{z \in \mathbf{C}^{N}\right.$ : $\left.\operatorname{Sup}_{\alpha}\left|a_{\alpha} z^{\alpha}\right|<\infty\right\}$. Moreover, the convergence is absolute and uniform on compact subsets of $\Omega$ so that the sum is analytic on $\Omega$.

LEMMA 2.1.4. If $\sigma$ is a class $K$ measure, then the domain of convergence, $\Omega$, of the power series $\Sigma_{\alpha \in Z_{+}^{N}}\left(z^{2 \alpha} / \hat{\sigma}(\alpha, \alpha)\right)$ is precisely $\Omega_{\sigma}$.

Proof. To see that $\Omega_{\sigma} \subset \Omega$, let $r$ be a point in $F_{\sigma}$. Let $z$ be a point of $\mathbf{C}^{N}$ which is dominated by $r$ in the sense that $\left|z_{j}\right|<\left|r_{j}\right|$ whenever $r_{j} \neq 0$. Let $\Lambda=\Lambda_{z}$ denote the set of integers $j$ for which $z_{j}=0$. Then

where $Z_{\Lambda}=\left\{\alpha \in Z_{+}^{N} \mid \alpha_{j}=0\right.$, for all $\left.j \in \Lambda\right\}$.

$$
\operatorname{Sup}_{\alpha} \frac{\left|z^{2 \alpha}\right|}{\hat{\sigma}(\alpha, \alpha)}=\operatorname{Sup}_{\alpha \in Z_{\Lambda}} \frac{\left|z^{2 \alpha}\right|}{\hat{\sigma}(\alpha, \alpha)}
$$


If $\alpha \in Z_{\Lambda}$, then $\hat{\sigma}(\alpha, \alpha)>\left|z^{2 \alpha}\right| \sigma\left(G_{z} \cap F_{\sigma}\right)$ where $G_{z}=\left\{v \in \mathbf{C}^{N}:\left|v_{j}\right|>\right.$ $\left|z_{j}\right|$, for all $\left.j \notin \Lambda\right\}$.

Since $r \in G_{z} \cap F_{\sigma}$, it follows from the above that

$$
\operatorname{Sup}_{\alpha} \frac{\left|z^{2 \alpha}\right|}{\hat{\sigma}(\alpha, \alpha)}<\frac{1}{\sigma\left(G_{z} \cap F_{\sigma}\right)}<\infty .
$$

Hence, by Theorem 2.1.3, $z \in \bar{\Omega}$ whenever $z$ is dominated by some $r$ in $F_{o}$, consequently $F_{\sigma} \subset \bar{\Omega}$.

Since $F_{o}$ is a compact, $T^{N}$-invariant subset of $\mathbf{C}^{N}$, it follows from Lemma 1 of $\left[12\right.$, p. 65] that the holomorphic hull of $F_{\sigma}$, namely $\bar{\Omega}_{\sigma}$, is the least log-convex Rinehardt set in $\mathbf{C}^{N}$ which contains $F_{\sigma}$.

Hence, $F_{\sigma} \subset \bar{\Omega}_{\sigma} \subset \bar{\Omega}$ and so $\Omega_{\sigma} \subset \Omega$.

Conversely, let $v \notin \bar{\Omega}_{\sigma}$.

Using Lemma 1 of $[12$, p. 65] once again, it is easily shown that there is an $\alpha \neq 0$ in $Z_{+}^{N}$ and an $\varepsilon=\varepsilon_{\alpha}>0$ such that $\left|v^{\alpha}\right|>(1+\varepsilon)^{|\alpha|} \cdot\left|z^{\alpha}\right|$ for all $z$ in $F_{\sigma}$.

With $\alpha$ fixed, choose $z=z_{\alpha}$ in $F_{\sigma}$ such that

$$
\left|z^{\alpha}\right|=\operatorname{Sup}_{u \in F_{o}}\left\{\left|u^{\alpha}\right|\right\}
$$

Then

$$
\frac{\left|v^{2 n \alpha}\right|}{\hat{\sigma}(n \alpha, n \alpha)}>\frac{\left[(1+\varepsilon)^{|\alpha|} \cdot\left|z^{\alpha}\right|\right]^{2 n}}{\left|z^{\alpha}\right|^{2 n} \cdot \sigma\left(F_{\sigma}\right)}=\frac{(1+\varepsilon)^{2 n|\alpha|}}{\sigma\left(F_{\sigma}\right)}
$$

for all $n=1,2, \ldots$.

Hence, by Theorem 2.1.3, $v \notin \bar{\Omega}$ which completes the proof of Lemma 2.1.4.

THEOREM 2.1.5. If $\sigma$ is of class $K$ and if $A$ is a compact subset of $\Omega_{\sigma}$, then the series $C_{\sigma}(z, w)=\sum_{\alpha \in Z_{+}^{N}}\left(\bar{z}^{\alpha} w^{\alpha} / \hat{\sigma}(\alpha, \alpha)\right)$ converges absolutely and uniformly on $\bar{\Omega}_{\sigma} \times A$.

Proof. Given $A$ a compact subset of $\Omega_{\sigma}$, choose $\lambda>1$ such that $\lambda A \subset \Omega_{\sigma}$.

If $(z, w) \in\left(\lambda \Omega_{\sigma}\right) \times\left((1 / \lambda) \Omega_{\sigma}\right)$, we have by Lemma 2.1.4 that

$$
\operatorname{Sup}_{\alpha}\left|[\hat{\sigma}(\alpha, \alpha)]^{-1}\left(\frac{1}{\lambda} z\right)^{2 \alpha}\right|=b_{z}<\infty
$$

and

$$
\operatorname{Sup}_{\alpha}\left|[\hat{\sigma}(\alpha, \alpha)]^{-1}(\lambda w)^{2 \alpha}\right|=b_{w}<\infty
$$

Since

$$
\left|z^{\alpha} \bar{w}^{\alpha}\right|=\left|\left(\frac{1}{\lambda} z\right)^{\alpha}\right| \cdot\left|(\lambda w)^{\alpha}\right|<\operatorname{Max}\left\{\left|\left(\frac{1}{\lambda} z\right)^{2 \alpha}\right|,\left|(\lambda w)^{2 \alpha}\right|\right\}
$$


it follows that

$$
\operatorname{Sup}_{\alpha}\left|\frac{z^{\alpha} \bar{w}^{\alpha}}{\hat{\sigma}(\alpha, \alpha)}\right| \leqslant \operatorname{Max}\left\{b_{z}, b_{w}\right\}<\infty .
$$

An application of Theorem 2.1.3 completes the proof.

Remarks. (a) Since $\sigma$ is $T^{N}$-invariant, the functions $[\hat{\sigma}(\alpha, \alpha)]^{-1 / 2} \cdot z^{\alpha}$ $\left(\alpha \in Z_{+}^{N}\right.$ ) form an orthonormal basis for a closed subspace $A^{2}(\sigma)$ of $L^{2}(\sigma)$. Consequently, $f(w)=\int f \cdot C_{\sigma}(\cdot, w) d \sigma\left(f \in A^{2}(\sigma), w \in \Omega_{\sigma}\right)$ (the basic ideas on reproducing kernels are given in [2]).

(b) The kernels

$$
C_{m_{s}}(z, w)=[1-\langle w, z\rangle]^{-N} \text { and } C_{m_{B}}(z, w)=[1-\langle w, z\rangle]^{-(N+1)}
$$

are known as the Szegő and Bergman kernels for $B$, respectively. In [3], Fefferman gives a detailed analysis of the Bergman kernel. The Szegő kernel is considered in [1].

(c) If $\sigma_{s}=h^{s} \cdot m_{B}$, where $h^{s}(z)=\left(1-|z|^{2}\right)^{s}(z \in B, s \in \mathbf{C}, \operatorname{Re} s>-1)$ then

$$
C_{\sigma_{s}}(z, w)=\left(\begin{array}{c}
N+s \\
N
\end{array}\right)[1-\langle w, z\rangle]^{-(N+1+s)}
$$

(The derivation and an application to bounded projections can be found in [10].)

THEOREM 2.1.6. Let $\sigma$ be a class $K$ measure satisfying $\sigma\left(\partial \Omega_{\sigma}\right)=0$. If $f \in L^{1}(\sigma)$ is holomorphic in $\Omega_{\sigma}$, then $f(w)=\int f \cdot C_{\sigma}(\cdot, w) d \sigma\left(w \in \Omega_{\sigma}\right)$.

Proof. For $0<r<1$, let $f_{r}$ be a dilation of $f, f_{r}(w)=f(r w)$.

If $f$ is holomorphic in $\Omega_{\sigma}$, then

$$
f_{r}(w)=\int f \cdot C_{\sigma}(\cdot, w) d \sigma \quad\left(w \in \Omega_{\sigma}\right)
$$

by remark (a).

If $\nu$ denotes Haar measure on the torus $T^{N}$, then [13, Theorem 3.4.2]

$$
\int\left|f_{r}(\omega w)\right| d \nu(\omega) \leqslant \int|f(\omega w)| d \nu(\omega)
$$

for $0<r<1$ and $w$ in $\Omega_{\sigma}$.

Integrating (2.2) (over $\Omega_{\sigma}$ ) with respect to $\sigma$, applying Fubini's theorem and then using the $T^{N}$-invariance of $\sigma$, leads to $\int\left|f_{r}\right| d \sigma \leqslant \int|f| d \sigma(0<r<1)$.

We may now apply Lebesgue's dominated convergence theorem to (2.1) to complete the proof.

\subsection{The kernels $K_{\sigma}$.}

Definition 2.2.1. Given $\sigma$, a class $K$ measure, define $K_{\sigma}: \bar{\Omega}_{\sigma} \times \Omega_{\sigma} \rightarrow(0, \infty)$ by $K_{\sigma}(z, w)=\left|C_{\sigma}(z, w)\right|^{2} / C_{\sigma}(w, w)$.

Concerning the kernels $K_{\sigma}$, we note that $K_{m_{B}}=\chi$ and $K_{m_{s}}=\beta$ as a consequence of remark (b). 
LEMMA 2.2.2. If $\sigma$ is a class $K$ measure, then $K_{\sigma}$ is continuous on $\bar{\Omega}_{\sigma} \times \Omega_{\sigma}$.

Proof. Follows directly from Theorem 2.1.5.

Definition 2.2.3. Given a class $K$ measure $\sigma$, with corresponding $\Omega_{\sigma}$ and $K_{\sigma}$, let $M\left(\bar{\Omega}_{\sigma}\right) \rightarrow^{T_{\sigma}} C^{\infty}\left(\Omega_{\sigma}\right)$ be given by $\left(T_{\sigma} \mu\right)(w)=\int K_{\sigma}(z, w) d \mu(z)$.

TheOREM 2.2.4. The maps $T_{\sigma}$ are one-to-one. That is, given a class $K$ measure $\sigma$, the map $T_{\sigma}: M\left(\bar{\Omega}_{\sigma}\right) \rightarrow C^{\infty}\left(\Omega_{\sigma}\right)$ is one-to-one.

Proof. As the map $T_{\sigma}$ is clearly linear, it suffices to show $T_{\sigma} \mu=0$ implies $\mu=0$.

For a fixed $w$ in $\Omega_{\sigma}$ and $\mu$ in $M\left(\bar{\Omega}_{\sigma}\right)$, it follows from Definitions 2.2.1 and 2.2.3 and Theorem 2.1.5 that

$$
\left(T_{\sigma} \mu\right)(w)=\left[C_{\sigma}(w, w)\right]^{-1} \sum_{\alpha} \sum_{\beta}[\hat{\sigma}(\alpha, \alpha) \hat{\sigma}(\beta, \beta)]^{-1}\left[\int z^{\alpha} \bar{z}^{\beta} d \mu\right] \bar{w}^{\alpha} w^{\beta}
$$

It is now clear that $T_{\sigma} \mu=0$ implies $\int z^{\alpha} \bar{z}^{\beta} d \mu=0$ for all $(\alpha, \beta) \in Z_{+}^{N} \times Z_{+}^{N}$. An application of the Stone-Weierstrass theorem completes the proof.

The following theorem is a direct consequence of Theorem 2.1.6 and Definition 2.2.1.

THEOREM 2.2.5. Let $\sigma$ be a class $K$ measure satisfying $\sigma\left(\partial \Omega_{\sigma}\right)=0$. If $f \in L^{1}(\sigma)$ is pluriharmonic in $\Omega_{\sigma}$, then $f(w)=\int f \cdot K_{\sigma}(\cdot, w) d \sigma\left(w \in \Omega_{\sigma}\right)$.

In Lemma 2.2.6 (below), $\left\{K_{s}\right\}_{s=0}^{\infty}$ denotes the Féjer kernel for $T$ and $\mu * K_{s}$ is the convolution of a measure $\mu$ with $K_{s}$ (for the specific definitions, see $[11, \S 1.1])$.

LeMMa 2.2.6. Let $\sigma$ be of class $K$ and let $\mu \in M\left(\bar{\Omega}_{\sigma}\right)$. If $T_{\sigma} \mu$ is pluriharmonic on $\Omega_{\sigma}$, then $T_{\sigma}\left(\mu * K_{s}\right)$ is a pluriharmonic polynomial.

Proof. Replace $\chi: \bar{B} \times B \rightarrow(0, \infty)$ by $K_{\sigma}: \bar{\Omega}_{\sigma} \times \Omega_{\sigma} \rightarrow(0, \infty)$ in the proof of Lemma 2.2 of [11].

THEOREM 2.2.7. Let $\sigma$ be of class $K$ and let $\mu \in M\left(\bar{\Omega}_{\sigma}\right)$. If $\sigma\left(\partial \Omega_{\sigma}\right)=0$ and if $T_{\sigma} \mu$ is pluriharmonic in $\Omega_{\sigma}$, then $T_{\sigma} \mu \in L^{1}(\sigma)$ and $\mu=\left(T_{\sigma} \mu\right) \cdot \sigma$.

Proof. Having established Lemma 2.2.2, Theorems 2.2.4 and 2.2.5 and Lemma 2.2.6, the proof of Theorem 2.2.7 is completed by the argument given in the proof of Theorem 2.3 of [11].

3. The spaces $H(\sigma)$ and $\operatorname{Re} H(\sigma)$. Let $H(\sigma)[\operatorname{Re} H(\sigma)]$ denote the $w\left[M\left(\bar{\Omega}_{\sigma}\right), C\left(\bar{\Omega}_{\sigma}\right)\right]$ closure of the class of all measures in $M\left(\bar{\Omega}_{\sigma}\right)$ of the form $g \sigma$ $[(\operatorname{Re} g) \sigma]$ where $g$ is a polynomial (see $[11, \S 3.1]$ for details).

The classical F. and M. Riesz theorem has been generalized as follows [7, Theorem 1.2].

TheOREM 3.1. If $\mu \in H(\sigma)$, then $\mu \ll \sigma$. 
For class $K$ measures, Theorem 3.1 can be phrased in terms of integral transforms.

THEOREM 3.2. If $\sigma$ is a class $K$ measure, and if $T_{\sigma} \mu$ is holomorphic in $\Omega_{\sigma}$, then $\mu \ll \sigma$.

Proof. If $T_{\sigma} \mu$ is holomorphic in $\Omega_{\sigma}$, then $T_{\sigma}\left(\mu * K_{s}\right)$ is a polynomial (recall the proof of Lemma 2.2.6).

Since $\mu * K_{s}$ converges weakly to $\mu$, it follows from Lemma 2.2.2 and Definition 2.2.3 that $\mu \in H(\sigma)$. Applying Theorem 3.1 completes the proof.

We shall now show that a theorem similar to 3.1 holds for the space $\operatorname{Re} H(\sigma)$.

THEOREM 3.3. Let $\sigma$ be a class $K$ measure satisfying $\sigma\left(\partial \Omega_{\sigma}\right)=0$ and let $f \in L^{1}(\sigma)$ be $T$-invariant and satisfy $|f| \geqslant \varepsilon>0$, a.e. $\sigma$. If $\mu \in \operatorname{Re} H(\sigma)$, then $T_{\sigma}(\mu / f)$ is pluriharmonic on $\Omega_{\sigma}$. Consequently, $\mu=T_{\sigma}(\mu / f) \cdot f \sigma$.

Proof. Having established Lemma 2.2.2 and Theorems 2.2.5 and 2.2.7, the proof of Theorem 3.2 is completed by the argument given in the proof of Theorem 3.3 of [11].

\section{BIBLIOGRAPHY}

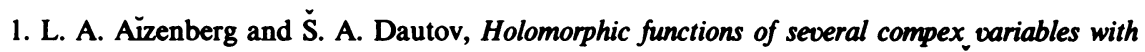
nonnegative real part. Traces of holomorphic and pluriharmonic functions on the Silov boundary, Math. USSR-Sb. 28 (1976), 301-313.

2. N. Aronszajn, Theory of reproducing kernels, Trans. Amer. Math. Soc. 68 (1950), 337-404.

3. C. Fefferman, The Bergman kernel and biholomorphic mappings of pseudo-convex domains, Invent. Math. 26 (1974), 1-66.

4. Frank Forelli, Measures whose Poisson integrals are pluriharmonic, Illinois J. Math. 18 (1974), 373-388.

5. __ Measures whose Poisson integrals are pluriharmonic. II, Illinois J. Math. 19 (1975), $584-592$.

6. __ Measures whose Poisson integrals are pluriharmonic. III (to appear).

7. _ The theorems of F. and M. Riesz for circular sets, Math. Scand. 33 (1973), 145-152.

8. F. Forelli and W. Rudin, Projections on spaces of holomorphic functions in balls, Indiana Univ. Math. J. 24 (1974), 593-602.

9. L. Hörmander, An introduction to complex analysis in several variables, Van Nostrand, Princeton, N. J., 1966.

10. C. Kolaski, A new look at a theorem of Forelli and Rudin, Indiana Univ. Math. J. (to appear).

11. , An F. and M. Riesz type theorem for the unit ball in complex $N$-space, Proc. Amer. Math. Soc. 61 (1976), 19-25.

12. L. Nachbin, Holomorphic functions, domains of holomorphy and local properties, American Elsevier, Inc., New York, 1970.

13. W. Rudin, Function theory in polydiscs, W. A. Benjamin, Inc., New York, 1969.

Department of Mathematics, University of Texas, San Antonio, Texas 78285 